\title{
METEORIZACIÓN E HIDROGEOQUÍMICA DE LOS RÍOS QUILISH Y PORCÓN EN LA CUENCA PORCÓN
}

\section{WHEATHERING AND HYDROGEOCHEMISTRY'S QUILISH AND PORCÓN RIVERS IN THE PORCÓN BASIN}

\author{
Carlos Malpica Sandoval ${ }^{a}$, Hugo Rivera Mantilla ${ }^{b}$, Sandra Rumay Villarreal ${ }^{a}$ y Víctor Vargas Rodríguez ${ }^{a}$
}

\section{RESUMEN}

Con los resultados obtenidos, podemos señalar que la meteorización produjo reacciones de hidrólisis, oxidación, disolución de silicatos, sulfuros y azufre originando minerales secundarios, iones y coloides (arcillas, $\mathrm{H}_{4} \mathrm{SiO}_{4}, \mathrm{SiO}_{2}$, $\mathrm{Fe}_{2} \mathrm{O}_{3}, \mathrm{FeOOH}, \mathrm{Fe}(\mathrm{OH})_{3}, \mathrm{Al}(\mathrm{OH})_{3}, \mathrm{Ca}^{2+}, \mathrm{Mg}^{2+}, \mathrm{Al}^{3+}, \mathrm{Fe}^{3+}, \mathrm{Na}^{+}, \mathrm{K}^{+}, \mathrm{SO}_{4}^{2-}, \mathrm{Pb}^{2+}, \mathrm{H}_{2} \mathrm{AsO}_{4}^{-}, \mathrm{Cu}^{2+}$ ), parte de los productos de la meteorización entran al sistema hidrológico y otra parte permanece en el suelo debido a su baja movilidad o por intercambio catiónico o aniónico; estas reacciones están determinadas por la energía libre, Eh y $\mathrm{pH}$. El análisis hidrogeoquímico se basa en la energía libre, constante de equilibrio, índice de saturación, diagramas $E h-p H, r N a / r C l$ y $\mathrm{rCl} / \mathrm{rHCO}_{3}^{-2}$, resultando agua de naturaleza cálcica-sódica, debido a la meteorización de los silicatos. Basándonos en los resultados obtenidos de los datos de energía libre determinamos que todas las reacciones están relacionadas con el índice de saturación, y basándonos en el diagrama Eh-pH, el hierro y aluminio reaccionan formando hidróxidos. Estudios petrológicos (30 muestras) y análisis hidrogeoquímico (9 muestras de agua), determinaron que los iones, coloides y moléculas originados por la meteorización de los silicatos y sulfuros que se encuentran en el río se deben a contaminación natural.

Palabras claves: Energía libre, índice de saturación, diagrama Eh-pH, disolución, hidrogeoquímica.

\begin{abstract}
With the results, we can point out that the wheathering produced reactions of hydrolyse, oxidation, silicate's dissolution, sulphurs and sulphur reactions; originating secondary minerals (clays, $\mathrm{H}_{4} \mathrm{SiO}_{4}, \mathrm{SiO}_{2}, \mathrm{Fe}_{2} \mathrm{O}_{3}, \mathrm{FeOOH}, \mathrm{Fe}(\mathrm{OH})_{3}, \mathrm{Al}(\mathrm{OH})_{3}$ $\mathrm{Ca}^{2+}, \mathrm{Mg}^{2+}, \mathrm{Al}^{3+}, \mathrm{Fe}^{3+}, \mathrm{Na}^{+}, \mathrm{K}^{+}, \mathrm{SO}_{4}^{2-}, \mathrm{Pb}^{2+}, \mathrm{H}_{2} \mathrm{AsO}_{4}^{-}, \mathrm{Cu}^{2+}$ ), part of the weathering products going to the hydrologic system, and another part remains in the soil due to its low mobility, or for cationic or anionic interchange; these reactions are determined by the free energy, Eh and $\mathrm{pH}$. The hydrochemistry analysis is based on the free energy, constant of balance, saturation index, graphs $\mathrm{Eh}-\mathrm{pH}, \mathrm{rNa} / \mathrm{rCl}$ and $\mathrm{rCl} / \mathrm{rHCO} 3-2 a n d$, standing out to be calc-sodic water, due to the silicates weathering.

Basing on the results obtained of the information of free energy, we determined that all the reactions are related to the saturation index and basing on the graph $\mathrm{Eh}-\mathrm{pH}$, the iron and aluminium react forming hydroxides. Petrologic studies (30 samples) and hydrogeochemistry analysis ( 9 water samples), determined that the ions, colloids and molecules that are in the river owe to natural pollution.
\end{abstract}

Key Words: Free energy, saturation index, Eh-pH diagram, dissolution, hydrogechemistry.

\section{INTRODUCCIÓN}

El área de estudio se centra en la subcuenca del río Porcón en cuya cabecera de cuenca está el cerro Quilish, zona en conflicto entre la empresa Minera Yanacocha y la comunidad del mismo nombre.

La composición química del agua está controlada por el clima (frío y húmedo con períodos secos), la litología (volcánicos, piroclastos y domos de flujo), procesos biológicos y la actividad antrópica.

\section{OBJETIVOS}

* Conocer las reacciones de meteorización que originan a los iones, moléculas y coloides a través del estudio hidrogeoquímico.

* Determinar de que forma (especiacion) las sustancias que se encuentran en el río son transportadas.

\footnotetext{
a Universidad Nacional de Cajamarca.

${ }^{\mathrm{b}}$ Universidad Nacional Mayor de San Marcos.
}

\section{HIPOTESIS}

El principal aporte de los contaminantes de la cuenca Porcón se debe a la meteorización que sufren las rocas que conforman la cuenca.

\section{MATERIALES Y METODOS}

\section{GEOGRAFÍA}

La subcuenca del río Porcón se ubica en el departamento de Cajamarca, en la cabecera de cuenca esta el cerro Quilish que alberga minerales metálicos.

El río Porcón es el receptor de las quebradas que drenan las laderas oeste y sur del cerro Quilish. Las aguas de la ladera este drenan por la quebrada Quilish, llamada aguas abajo como río Chilincaga. La ladera sur es drenada por el río Quilish. Ambas quebradas se unen 
aproximadamente a $8 \mathrm{~km}$ al sur del cerro Quilish para formar el río Porcón, que se une aguas abajo al río Grande (Fig. 1). Estas aguas sirven para el consumo humano de la ciudad de Cajamarca.

\section{GEOLOGÍA}

Los procesos tectónicos y volcánicos muy activos de la cordillera de los Andes son los que causan su formación. Una secuencia de flujos silicios o lavas ricas en ácidos fluyeron a lo largo de los principales ductos estructurales dando lugar a una serie de estructuras de domos de flujo, formando parte de un cinturón volcánico que se extiende desde Cajamarca hasta la frontera ecuatoriana, a $350 \mathrm{~km}$. Al noroeste estas rocas han sido localmente silicificadas y mineralizadas por soluciones hidrotermales ricas en oro, hay fracturas rellenas por azufre nativo y hematita.

Está formada por rocas volcánicas de la era terciaria que recubren un basamento cretáceo. Las rocas volcánicas terciarias incluyen piroclástos, tobas y andesitas.

En nuestra zona predominan andesitas con fenocristales de plagioclasas, su matriz tienen diferente grado de meteorización a arcillas y sericita; los piroxenos y anfíboles están como fenocristales alterados a cloritas. En las inmediaciones del río Chilincaga observamos zonas de alteración argílica fracturadas rellenas con azufre nativo y hematita (INGEMMET, MWH American).

\section{PROCESO DE METEORIZACIÓN}

La meteorización, como proceso de cambios físicos y químicos de los minerales y rocas en ambientes superficiales por acción de los agentes atmosféricos (ambiente geoquímico secundario); actúa para alcanzar el equilibrio entre los minerales primarios con las condiciones prevalecientes en el nuevo ambiente

\section{Condiciones Químicas en Superficie}

Disolución del $\mathrm{CO}_{2}$ y $\mathrm{O}_{2}$ en la Atmósfera

El proceso de disolución del $\mathrm{CO}_{2(g)}$ en el agua es:

$$
\begin{gathered}
\mathrm{CO}_{2(\mathrm{~g})} \leftrightarrow \mathrm{CO}_{2(\mathrm{ac})} \\
\mathrm{CO}_{2(\mathrm{ac})}+\mathrm{H}_{2} \mathrm{O} \leftrightarrow \mathrm{H}_{2} \mathrm{CO}_{3(\mathrm{ac})} \\
\mathrm{H}_{2} \mathrm{CO}_{3} \leftrightarrow \mathrm{H}^{+}+\mathrm{HCO}_{3}^{-} \\
\mathrm{HCO}_{3}^{-} \leftrightarrow \mathrm{H}^{+}+\mathrm{CO}_{3}^{2-}
\end{gathered}
$$

La solubilidad del gas aumenta todavía más cuando ocurre una reacción entre el $\mathrm{CO}_{2}$ y el $\mathrm{H}_{2} \mathrm{O}$.

De los datos del SENAMHI-Cajamarca en la atmósfera hay $0.033 \%$ de $\mathrm{CO}_{2}(\mathrm{~g})$, a la temperatura máxima de $21,4^{\circ} \mathrm{C}$, le da un $\mathrm{pH}$ de 5,6 ; a la temperatura mínima de $7,2^{\circ} \mathrm{C}$ le da un $\mathrm{pH}$ de 5,5 a la lluvia y al vapor de agua.

De los mismos datos del SENAMHI-Cajamarca, en la atmósfera hay $21 \%$ de $\mathrm{O}_{2}(\mathrm{~g})$ que a la temperatura máxima de $21,4^{\circ} \mathrm{C}$ la concentración de saturación del $\mathrm{O}_{2}(\mathrm{~g})$ es $9,5 \mathrm{mg} / \mathrm{l}$, a la temperatura mínima de $7,2^{\circ} \mathrm{C}$ la concentración de saturación del $\mathrm{O}_{2}(\mathrm{~g})$ es $12,18 \mathrm{mg} / \mathrm{l}$. en la lluvia y el vapor de agua. Las condiciones atmosféricas imperantes, la lluvia y el vapor de agua son siempre ácidos y oxidantes.

\section{METEORIZACIÓN EN LA SUBCUENCA}

El proceso de meteorización produce una zona de oxidación-lixiviación, donde se originan la hidrólisis, hidratación y disolución.

La meteorización de los minerales metálicos se debe a que el agua de lluvia o vapor de agua son oxidantes formando una solución agua - oxígeno - anhídrido carbónico, el cual penetra y oxida a los sulfuros, liberando los cationes metálicos y anión sulfato, estos pueden ser transportados en solución superficiales desde el lugar de oxidación a otro lugar, o penetrar en profundidad.

Para los silicatos, el proceso es el mismo, la meteorización cambia a los minerales primarios densos de las rocas por minerales pequeños, porosos y menos densos (en nuestro caso, arcillas, iones y moléculas) los cuales difieren en composición química y estructural de los minerales originarios.

\section{Modelo de Generación de Minerales Supérgenos Pirita a hematita}

$2 \mathrm{FeS}_{2}+8,5 \mathrm{O}_{2}+2 \mathrm{H}_{2} \mathrm{O} \leftrightarrow \mathrm{Fe}_{2} \mathrm{O}_{3}+4 \mathrm{SO}_{4}^{2-}+4 \mathrm{H}^{+}$

\section{Pirita a goethita}

$2 \mathrm{FeS}_{2}+7 \mathrm{O}_{2}+6 \mathrm{H}_{2} \mathrm{O} \leftrightarrow \mathrm{FeOOH}+4 \mathrm{SO}_{4}^{2-}+10 \mathrm{H}^{+}$

Azufre

$\mathrm{S}_{2}+2 \mathrm{H}_{2} \mathrm{O}+3 \mathrm{O}_{2} \leftrightarrow 2 \mathrm{H}_{2} \mathrm{SO}_{4}$

Reacción de disolución de silicatos produce neutralización

Plagioclasa arcillas

$$
\begin{aligned}
& \mathrm{CaAl}_{2} \mathrm{Si}_{2} \mathrm{O}_{8}+4 \mathrm{H}^{+}+1 / 2 \mathrm{O}_{2} \leftrightarrow \mathrm{Al}_{2} \mathrm{Si}_{2} \mathrm{O}_{5}(\mathrm{OH})_{4}+\mathrm{Ca}^{2+} \\
& \mathrm{Na}_{2} \mathrm{AlSi}_{3} \mathrm{O}_{8}+4 \mathrm{H}^{+}+1 / 2 \mathrm{O}_{2} \leftrightarrow \mathrm{Al}_{2} \mathrm{Si}_{2} \mathrm{O}_{5}(\mathrm{OH})_{4}+\mathrm{Ca}^{+} 4 \mathrm{SiO}_{2}
\end{aligned}
$$

\section{Feldespato potásico - Sericita}

$3 \mathrm{KAISi}_{3} \mathrm{O}_{8}+2 \mathrm{H}^{+}+12 \mathrm{H}_{2} \mathrm{O} \leftrightarrow \mathrm{KAl}_{3} \mathrm{Si}_{3} \mathrm{O}_{8}(\mathrm{OH})_{2}+6 \mathrm{H}_{4} \mathrm{SiO}_{4}$ $+2 \mathrm{~K}^{+}$

\section{Ácido ortosilícico a sílice amorfa}

$\mathrm{H}_{4} \mathrm{SiO}_{4} \leftrightarrow \mathrm{SiO}_{2(\mathrm{am})}+2 \mathrm{H}_{2} \mathrm{O}$

\section{HIDROGEOQUÍMICA}

\section{Muestras de agua}

Los datos son de la Mesa de Diálogo de Cajamarca entre la Minera y la Comunidad 2004-2005. Para el análisis se escogieron nueve (9) muestras tomadas en el mes de Marzo y nueve (9) del mes de Julio (Tabla 1). 
Quilish: muestra 1 (QQUI-1) y 2 (QQUI-2)

- Porcón las muestras 3 (RPPM-2), 5 (RPPM-3) y 6 (RPO)

- Río Chilincaga la muestra 4 (RCHIL)

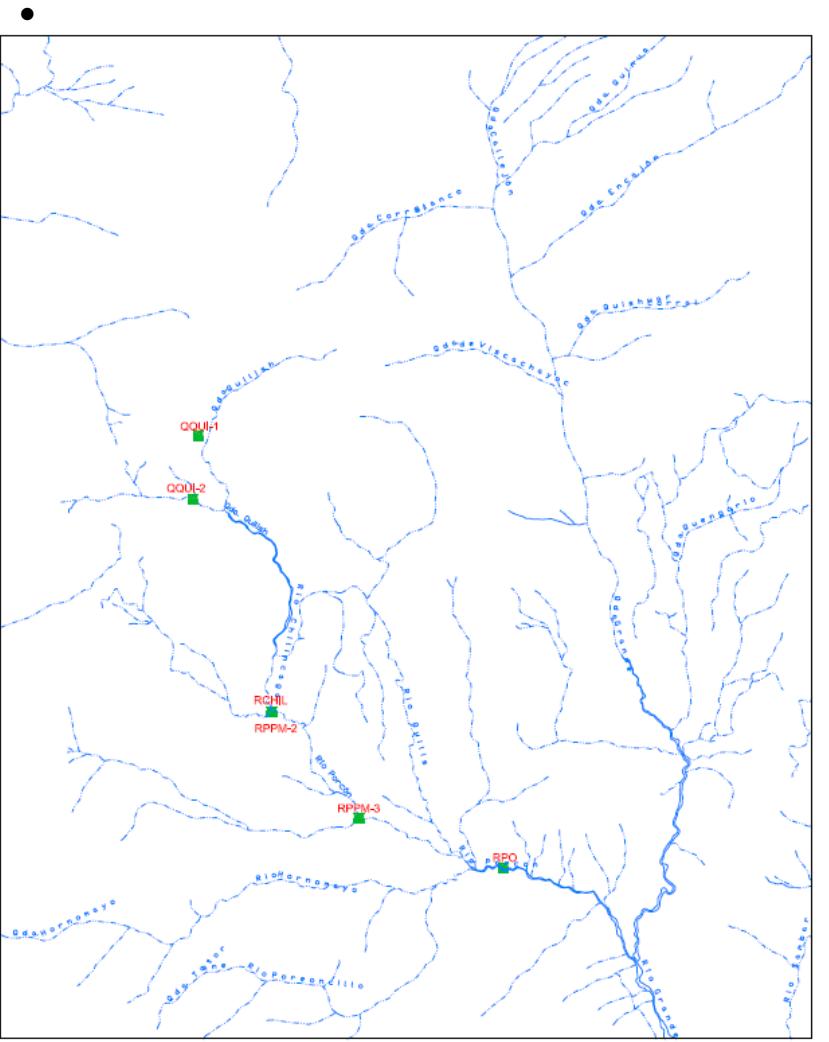

Fig.1 Mapa hidrográfico y de toma de muestras de la subcuenca Porcón

\section{Análisis Hidrogeoquímico}

El transporte de un elemento químico por el drenaje superficial (sistema hidrológico) puede ocurrir bajo varias formas (Nesbitt, 1984). Por termodinámica y geoquímica, es posible evaluar en principio la probable distribución de los diferentes elementos entre las diversas formas indicadas. molecular (minerales), iones complejos, coloides y en partículas finas en suspensión

\section{Posibles Reacciones Químicas Producidas en el Río}

Formación del ión arsenato:

$\mathrm{H}_{2} \mathrm{AsO}_{4}^{-}+\mathrm{H}^{+} \leftrightarrow \mathrm{H}_{3} \mathrm{AsO}_{4}$

$\mathrm{SI}=$ entre 13.38 a 8.92 . En las condiciones que se tomaron las muestras la fase estable es $\mathrm{H}_{2} \mathrm{AsO}_{4}^{-}$,

Formación del ión arsenito:

$\mathrm{H}_{2} \mathrm{AsO}_{4}^{-} \leftrightarrow \mathrm{H}^{+}+\mathrm{HAsO}_{4}^{2-}$

$\mathrm{SI}=$ entre $-8.81 \mathrm{a}-4.35$

Se forma $\mathrm{HAsO}_{4}^{2-}$ y este cambio se debe a la variación del $\mathrm{pH}$ que sufre el agua.

Formación del sulfato:

$\mathrm{HSO}_{4}^{-} \leftrightarrow \mathrm{H}^{+}+\mathrm{SO}_{4}^{2-}$

$\mathrm{SI}=$ entre -9.16 a -7.84
Los datos de SI y del diagrama Eh-pH demuestran que la fase estable es $\mathrm{SO}_{4}^{2-}$, Formación del ácido carbónico:

$\mathrm{H}_{2} \mathrm{CO}_{3} \leftrightarrow \mathrm{H}^{+}+\mathrm{HCO}_{3}^{-}$

$\mathrm{SI}=$ entre $-4.51 \mathrm{a}-4.06$

En las condiciones que se obtuvieron las muestras si se forma el $\mathrm{H}_{2} \mathrm{CO}_{3}$.

Formación del bicarbonato:

$\mathrm{HCO}_{3}^{-} \leftrightarrow \mathrm{H}+\mathrm{CO}_{3}^{2-}$

$\mathrm{SI}=$ entre $-4,37$ a -3.30

En las condiciones que se obtuvieron las muestras si se forma el $\mathrm{HCO}_{3}^{-}$, la diferencia con las muestras 1 y 2 es el cambio de $\mathrm{pH}$ lo que ocasiona su formación.

Formación del hidróxido de aluminio:

$\mathrm{Al}(\mathrm{OH})_{3} \leftrightarrow \mathrm{Al}^{3+}+3 \mathrm{OH}^{-}$

$\mathrm{SI}=$ entre 8.35 a 3.34

$\mathrm{El} \mathrm{Al}(\mathrm{OH})_{3}$ se forma y es estable en cualquier parte del río.

Formación del hidróxido de hierro:

$\mathrm{Fe}(\mathrm{OH})_{3} \leftrightarrow \mathrm{Fe}^{3+}+\mathrm{OH}^{-}$

$\mathrm{SI}=$ entre 5.46 a 0.70

$\mathrm{El} \mathrm{Fe}(\mathrm{OH})_{3}$ se forma y es estable en cualquier parte del río .

Formación de sílice amorfa:

$\mathrm{SiO}_{2}(\mathrm{am})+2 \mathrm{H}_{2} \mathrm{O} \leftrightarrow \mathrm{H}_{4} \mathrm{SiO}_{4}$

$\mathrm{SI}=\mathrm{Na}$ hay datos en el informe

Diagrama Eh-pH: En todas las muestras la fase estable es $\mathrm{SiO}_{2}(\mathrm{am})$.

Los coloides permiten la migración de cationes y aniones que bajo determinadas condiciones de Eh-pH no sería posible; un ejemplo es el arsénico, que puede migrar como aniones complejos de arsenito o arsenato, en un amplísimo rango de condiciones Eh-pH (Brookim D. 1988). En presencia de coloides son adsorbidos neutralizando al coloide y precipitándose como gel.

El hierro no puede migrar como $\mathrm{Fe}^{3+}$ pero si puede hacerlo como finas partículas de goethita.

La adsorción del plomo puede ser descrita como: FeO $\mathrm{OH}+\mathrm{Pb}^{2+} \leftrightarrow \mathrm{FeOPb}+\mathrm{H}^{+}$, esto es importante en lo que se refiere a la fijación del plomo lixiviado:

$\mathrm{PbS}+\mathrm{H}_{2} \mathrm{CO}_{3}^{-}+2 \mathrm{O}_{2} \leftrightarrow \mathrm{Pb}^{2+}+2 \mathrm{H}_{2} \mathrm{CO}_{3}^{-}+\mathrm{SO}_{4}^{2-}+$

$2 \mathrm{H}^{+}$

El plomo así lixiviado puede migrar en $\mathrm{pH}$ ácido, pero a medida que éste sube, el plomo va quedando como catión adsorbido en goethita, cuando supera un $\mathrm{pH}$ de 6 , todo el plomo ha sido retenido (Higueras $P . y$ Oyarzun R.).

Según las proporciones $\mathrm{rNa} / \mathrm{Cl}$ y $\mathrm{rCl} / \mathrm{rHCO}_{3}^{-}$(Ver Tabla № 1) se puede afirmar que la composición química de las aguas del río es consecuencia de la meteorización de los silicatos. (Custodio, 1983). 


\section{CONCLUSIONES}

- La meteorización de los minerales de las rocas en la subcuenca es un proceso natural, sus reacciones químicas regulan el Eh y $\mathrm{pH}$ de las aguas y cuyos productos resultantes son transportados $\mathrm{e}$ incorporados al sistema hidrológico de la zona.

- Del análisis hidrogeoquímico se determinó que el agua es de naturaleza cálcica-sódica, y las únicas reacciones que se producen en el río son las de los elementos: aluminio, hierro y arsénico, ellos pueden ser transportados en suspensión o depositarse por precipitación.
Al formarse el arsenito, arsenato y el ión plomo, parte de estos elementos tóxicos son transportados por el río y la otra parte se encuentra en los sedimentos, al ser adsorbidos y precipitados por los coloides.

- hidrogeoquímica determinan que se forma la goethita, entonces hay la posibilidad que en los sedimentos se encuentre plomo y arsénico; por lo que sería necesario hacer tales estudios.

\section{AGRADECIMIENTO}

Agradecemos al Ing. David Gómez Hidalgo. por el apoyo brindado.

TABLA № 1: ANÁLISIS QUÍMICO Y RELACIONES HIDROGEOQUÍMICAS DEL AGUA DE LOS RÍOS QUILISH, PORCÓN Y CHILINCAGA

\begin{tabular}{|c|c|c|c|c|c|c|c|c|c|c|c|c|}
\hline Muestra & 1 & & 2 & & 3 & & 4 & & 5 & & T & \\
\hline Aniones & $\mathrm{meg} / \mathrm{l}$ & \begin{tabular}{|l|} 
act \\
$(\mathrm{mol} / \mathrm{l})$
\end{tabular} & $\mathrm{meg} / \mathrm{l}$ & act $(\mathrm{mol} / \mathrm{l})$ & $\mathrm{meg} / \mathrm{l}$ & \begin{tabular}{|l|} 
act \\
$(\mathrm{mol} / \mathrm{l})$
\end{tabular} & $\mathrm{meg} / \mathrm{l}$ & act $(\mathrm{mol} / \mathrm{l})$ & $\mathrm{meg} / \mathrm{l}$ & act (mol/l) & $\mathrm{meg} / \mathrm{l}$ & act (mol/l) \\
\hline $\mathrm{HCO}^{-2}{ }_{3}$ & 0.05 & $4.55 \times 10^{-5}$ & 0.03 & $2.44 \times 10^{-5}$ & 0.14 & $1.32 \times 10^{-4}$ & 0.21 & $1.92 \times 10^{-4}$ & 0.14 & $1.34 \times 10^{-4}$ & 0.56 & $5.33 \times 10^{-4}$ \\
\hline $\mathrm{SO}^{-2} 4$ & 0.12 & $5.34 \times 10^{-5}$ & 0.35 & $1.57 \times 10^{-4}$ & 1.39 & $5.70 \times 10^{-5}$ & 2.4 & $8.62 \times 10^{-4}$ & 0.98 & $3.88 \times 10^{-4}$ & 0.8 & $3.23 \times 10^{-4}$ \\
\hline $\mathrm{CO}^{-2}{ }_{3}$ & $7 \times 10^{-6}$ & $3.6 \times 10^{-4}$ & $3 \times 10^{-6}$ & $1.31 \times 10^{-9}$ & $9 \times 10^{-5}$ & $3.83 \times 10^{-8}$ & $9 \times 10^{-5}$ & $3.49 \times 10^{-5}$ & $2 \times 10-4$ & $7.85 \times 10^{-8}$ & 0.002 & $9.51 \times 10^{-7}$ \\
\hline $\mathrm{Cl}^{-}$ & 0.004 & $4.1 \times 10^{-6}$ & \begin{tabular}{|l|}
0.01 \\
\end{tabular} & $6.59 \times 10^{-6}$ & \begin{tabular}{|l|}
0.06 \\
\end{tabular} & $5.24 \times 10^{-5}$ & 0.1 & $9.27 \times 10^{-5}$ & \begin{tabular}{|l|}
0.03 \\
\end{tabular} & $2.86 \times 10^{-5}$ & 0.04 & $3.94 \times 10^{-5}$ \\
\hline Suma & 0.17 & $1.03 \times 10^{-4}$ & 0.39 & $1.93 \times 10^{-4}$ & 1.59 & $2.41 \times 10^{-4}$ & 2.71 & 0.001 & 1.15 & $5.51 \times 10^{-4}$ & 1.4 & $8.96 \times 10^{-4}$ \\
\hline Cationes & & & & & & & & & & & & \\
\hline $\mathrm{Ca}$ & 0.1 & $4.60 \times 10^{-5}$ & 0.24 & $1.05 \times 10^{-4}$ & 0.93 & $3.79 \times 10^{-4}$ & 3.34 & $1.13 \times 10^{-3}$ & 0.65 & $2.57 \times 10^{-4}$ & 1.06 & $4.28 \times 10^{-4}$ \\
\hline $\mathrm{Na}$ & 0.04 & $3.80 \times 10^{-5}$ & 0.1 & $9.62 \times 10^{-5}$ & 0.32 & $3.10 \times 10^{-4}$ & 0.54 & $5.16 \times 10^{-4}$ & 0.22 & $2.11 \times 10^{-4}$ & 0.25 & $1.44 \times 10^{-4}$ \\
\hline $\mathrm{Fe}$ & 0.01 & $2.60 \times 10^{-6}$ & 0.01 & $2.54 \times 10^{-6}$ & 0.07 & $2.37 \times 10^{-4}$ & 0.1 & $2.43 \times 10^{-5}$ & 0.04 & $1.26 \times 10^{-5}$ & 0.05 & $1.54 \times 10^{-5}$ \\
\hline $\mathrm{K}$ & 0.01 & $8.80 \times 10^{-6}$ & 0.01 & $1.54 \times 10^{-5}$ & 0.05 & $4.28 \times 10^{-5}$ & 0.07 & $6.07 \times 10^{-5}$ & 0.03 & $2.92 \times 10^{-5}$ & 0.04 & $3.92 \times 10^{-5}$ \\
\hline Al & 0.02 & $4.60 \times 10^{-6}$ & 0.16 & $1.31 \times 10^{-4}$ & 0.18 & $3.84 \times 10^{-5}$ & 0.26 & $4.33 \times 10^{-5}$ & 0.16 & $3.38 \times 10^{-5}$ & 0.16 & $3.43 \times 10^{-5}$ \\
\hline Suma & 0.18 & $1.00 \times 10^{-4}$ & 0.52 & $2.32 \times 10^{-4}$ & 1.55 & $1.01 \times 10^{-3}$ & 4.31 & 0.002 & 1.1 & $5.44 \times 10^{-4}$ & 1.56 & $6.61 \times 10^{-4}$ \\
\hline $\mathrm{T}^{\circ} \mathrm{C}$ & 13.7 & & 14.0 & & 13.7 & & 13.7 & & 13.8 & & 17.8 & \\
\hline $\mathrm{pH}$ & 6.24 & & 6.01 & & 6.85 & & 6.69 & & 7.17 & & 7.64 & \\
\hline S.D. & 2.3 & & 30 & & 96 & & 149 & & 72 & & 89 & \\
\hline S.S & 5 & & 6 & & 22 & & 30 & & 22 & & 25 & \\
\hline $\mathrm{Pb}$ & 0.001 & $4.48 \times 10^{-9}$ & 0.001 & & 0.001 & $3.95 \times 10^{-9}$ & 0.001 & & 0.001 & $3.80 \times 10^{-9}$ & 0.001 & $3.88 \times 10^{-9}$ \\
\hline As & 0.001 & $1.23 \times 10^{-8}$ & 0.001 & & 0.001 & $1.09 \times 10^{-8}$ & 0.001 & & 0.001 & $1.05 \times 10^{-8}$ & 0.001 & $1.07 \times 10^{-8}$ \\
\hline $\mathrm{H}$ & & $5.64 \times 10^{-7}$ & & & & $2.51 \times 10^{-7}$ & & & & $6.40 \times 10^{-8}$ & & $2.18 \times 10^{-8}$ \\
\hline $\begin{array}{l}\mathrm{rCl} / \\
\mathrm{rHCO}^{-2}{ }_{3}\end{array}$ & 0.09 & & 0.22 & & 0.4 & & 0.49 & & 0.21 & & 0.073 & \\
\hline $\mathrm{rNa} / \mathrm{rCl}$ & \begin{tabular}{|l|}
8.81 \\
\end{tabular} & & 14.39 & & 5,67 & & 5.33 & & $\begin{array}{l}, 21 \\
7.07\end{array}$ & & \begin{tabular}{|l|}
6.17 \\
\end{tabular} & \\
\hline $\mathrm{rCa} / \mathrm{rNa}$ & 2.67 & & 2.42 & & 2.97 & & 5.83 & & 3.04 & & 4.2 & \\
\hline Tipo agua & Ca-Na & & Ca-Na & & Ca-Na & & Ca-Na & & Ca-Na & & Ca-Na & \\
\hline $\begin{array}{l}\text { Fuerza } \\
\text { iónica }\end{array}$ & 0.0003 & & 0.0008 & & 0.0022 & & 0.0066 & & 0.0031 & & 0.0026 & \\
\hline
\end{tabular}

\section{REFERENCIAS BIBLIOGRAFICAS}

1. Custodio E, Llamas M. R..(1983).- Hidrología Subterránea. Ed. Omega. Barcelona, 2350p.

2. Higueras, P. y Oyarzun, R. Curso de mineralogía y geoquímica ambiental. Internet, 57p.

3. Informe 2004-2005 de la Mesa de Diálogo de Cajamarca.

4. INGEMMET (1980).- Cuadrángulo de Cajamarca (15-f) Boletín Serie A: Carta Geológica Nacional No. 31, Lima.

5. M W H American INC (2002).- Estudio de impacto ambiental proyecto cerro Negro, Vol I

6. Nesbitt, H. W. (1984).- Equilibrium diagram displaying chemical speciation and mineral stabilities in aqueous solutions in Environmental Geochemistry (M. E. Fleet, ed.) .Mineralogical Association of Canada, Ontario, Short Course Handbook No.10, 15-44p.

7. SENAMHI (2006).- Servicio Nacional de Meteorología e Hidrología. Dirección regional de Cajamarca.

e-mail: cmalpicas@hotmail.com y hriveram@speedy.com.pe 